\title{
Sclerotherapy for Venous Malformations of Head and Neck: Systematic Review and Meta-Analysis
}

Lucio De Maria, $\mathrm{MD}^{1}$, Paolo De Sanctis, $\mathrm{MS}^{2}$, Karthik Balakrishnan, MD, $\mathrm{MPH}^{3}$, Megha Tollefson, $\mathrm{MD}^{4}$, Waleed Brinjikji, $\mathrm{MD}^{5}$

${ }^{1}$ Department of Neurosurgery, Mayo Clinic, Rochester, MN, USA

${ }^{2}$ Humanitas University, Milano, IT, USA

${ }^{3}$ Department of Otorhinolaryngology, Mayo Clinic, Rochester, MN, USA

${ }^{4}$ Department of Dermatology and Pediatrics, Mayo Clinic, Rochester, MN, USA

${ }^{5}$ Department of Radiology and Vascular Centers, Mayo Clinic, Rochester, MN, USA

We performed a systematic review and meta-analysis of studies performing sclerotherapy for treatment of venous malformations (VMs) of the face, head and neck. It is our hope that data from this study could be used to better inform providers and patients regarding the benefits and risks of percutaneous sclerotherapy for treatment of face, head and neck VMs. We searched PubMed, MEDLINE, and EMBASE from 2000-2018 for studies evaluating the safety and efficacy of percutaneous sclerotherapy of neck, face and head VMs. Two independent reviewers selected studies and abstracted data. The primary outcomes were complete and partial resolution of the VM. Data were analyzed using random-effects meta-analysis. Thirty-seven studies reporting on 2,067 patients were included. The overall rate of complete cure following percutaneous sclerotherapy with any agent was $64.7 \%$ (95\% confidence interval [Cl], 57.4-72.0\%). Sodium tetradecyl sulfate had the lowest complete cure rate at $55.5 \%(95 \% \mathrm{Cl}, 36.1-74.9 \%)$ while pingyangmycin had the highest cure rate at $82.9 \%(95 \% \mathrm{Cl}, 71.1-94.7 \%)$. Overall patient satisfaction rates were $91.0 \%$ (95\% Cl, 86.1-95.9\%). Overall quality of life improvement was $78.9 \%(95 \% \mathrm{Cl}$, $67.0-90.8 \%)$. Overall permanent morbidity/mortality was $0.8 \%(95 \% \mathrm{Cl}, 0.3-1.3 \%)$ with no cases of mortality. Our systematic review and meta-analysis of 37 studies and over 2,000 patients found that percutaneous sclerotherapy is a very safe and effective treatment modality for treatment of VMs of the head, neck and face.

Key Words: Venous malformations; Venous; Head and neck; Sclerotherapy

\section{INTRODUCTION}

Venous malformations (VMs) are slow flow developmental anomalies of the veins which do not proliferate and normally do not involute. ${ }^{1-37}$ These lesions can develop anywhere in the body, including structures of the face, head and neck. Due to the delicate interplay be- tween aesthetics, function, and anatomy in this region, management of these lesions with any treatment modality can be challenging.

Over the past several decades, sclerotherapy with various agents has been demonstrated to be effective for face, head and neck VMs. ${ }^{1,6,13,38-40}$ However, the evidence for treatment of these

\section{Correspondence to:} Lucio De Maria, MD

Department of Neurosurgery, Mayo

Clinic, 200 First St. SW, Rochester, MN 55905, USA

Tel: +393495937422

Fax: +1 5072840688

E-mail: luciodemaria@libero.it

Received: September 2, 2019

Revised: November 23, 2019

Accepted: December 23, 2019
Copyright $\odot 2020$ Korean Society of Interventional Neuroradiology

This is an Open Access article distributed under the terms of the Creative Commons Attribution Non-Commercial License (http://creativecommons.org/licenses/by-nc/3.0) which permits unrestricted non-commercial use, distribution, and reproduction in any medium, provided the original work is properly cited.

pISSN 2093-9043 eISSN 2233-6273 
lesions is often based off of smaller case series which makes generalizing results to the greater population difficult. Furthermore, comparative studies examining the efficacy and safety of various sclerotherapy agents are few and far between. The goals of this systematic review and meta-analysis were to 1) understand the overall safety and efficacy rates of percutaneous sclerotherapy for treatment of VMs in the face, head and neck and 2) compare safety and efficacy rates of commonly used sclerotherapy agents. It is our hope that data from this study could be used to better inform providers and patients regarding the benefits and risks of percutaneous sclerotherapy for treatment of face, head and neck VMs.

\section{MATERIALS AND METHODS}

\section{Literature search}

The systematic review is reported according to the Preferred Reporting Items for Systematic Reviews and Meta-Analysis guidelines. ${ }^{37}$ A comprehensive literature search of the databases PubMed, Ovid MEDLINE, and Ovid EMBASE was designed and conducted by an experienced librarian with input from the authors. The search duration was 2 months. The key words "sclerotherapy," "vascular malformations," "venous malformations," "arteriovenous malformation," "hemangioma," "lymphatic malformation, "head," "neck," "facial," "oropharyngeal," and "orbital" were used in "AND" and "OR" combinations. The search was limited to articles published from 2000 to 2018. Inclusion criteria were the following: 1) English or Italian language, 2) case series reporting greater than 5 patients, 3) studies reporting image guided percutaneous sclerotherapy, 4) studies reporting exclusively face, head and neck VMs or subdividing outcomes and complications by anatomical region, and 5) studies classifying VMs appropriately using the International Society for the Study of Vascular Anomalies. Exclusion criteria were: 1) case series reporting fewer than 5 patients, 2) case reports, 3) vascular malformation not of the head and/or neck region (e.g., sclerotherapy for varicose veins in legs), and 4) studies not classifying lesions according to the International Society for the Study of Vascular Anomalies criteria. International Society for the Study of Vascular Anomalies criteria on imaging and clinical exam for VMs on imaging include 1) septated lobulated T2 hyperintense and T1 hypointense mass without mass effect, 2) phleboliths which are characteristically hypointense on $T 1 / T 2$, 3) presence of fluid-fluid levels, 4) no flow voids on spin echo sequences, 5) the lesion infiltrates tissue planes, 6) no arterial or early venous enhancement, and 7) diffuse enhancement on delayed images. ${ }^{41}$ On clinical exam, VMs appear as faint blue, soft and easily compressible non-pulsatile masses. The lesions characteristically enlarged with Valsalva maneuver and in dependent positions and decompress with local compression.

In studies with overlapping patient populations written by the same author/institution, we only included the largest or most complete dataset. In cases where outcomes were separated out by the type of sclerotherapy agent used, we abstracted outcomes separately for each agent in order to perform our subgroup analyses. Two authors determined inclusion and exclusion criteria for the studies in the literature search with differences resolved by the senior author.

\section{Outcomes and data extraction}

For each study, we extracted the following baseline information: number of patients, mean or median age and gender, number of malformations treated, location of malformations, sclerosing agent and its mean volume used, mean number of treatment sessions, and mean length of radiographic and clinical follow-up. The primary outcome of this study is the efficacy of sclerotherapy which includes complete cure of the vascular malformation (resolution of the VM on physical exam), partial cure of the vascular malformation (partial decrease in VM size), lack of benefit following sclerotherapy, improvement in quality of life (QoL), and patient satisfaction. Secondary outcomes are adverse events after sclerotherapy, including respiratory complications, skin necrosis/scars, any permanent morbidity/mortality, local temporary complications). Permanent morbidity and mortality were defined as mortality or any permanent neurological deficit. Local temporary complications included erythema, swelling, and pain.

For our subgroup analysis by sclerotherapy agent, we separated outcomes by agent. We were able to abstract data for the following individual agents: bleomycin, ethanol, sodium tetradecyl sulfate (STS), ethanolamine and pingamycin.

\section{Study risk of bias}

We modified the Newcastle-Ottawa Quality Assessment Scale to assess the methodologic quality of the studies included in this meta-analysis. This tool is designed for use in comparative studies; however, because the studies did not include a control group, we assessed study risk of bias 
based on selected items from the tool, focusing on the following questions: 1) Did the study include all patients or consecutive patients versus a selected sample?, 2) Was the study retrospective or prospective?, 3) Was clinical follow-up satisfactory, thus allowing ascertainment of all outcomes? 4) Were outcomes clearly reported?, and 5) Were there clearly defined inclusion and exclusion criteria?

\section{Statistical analysis}

We estimated from each cohort the cumulative prevalence and $95 \%$ confidence interval (Cl) for each outcome. Event rates were pooled across studies with a random-effects meta-analysis. Heterogeneity across studies was evaluated using the $\mathrm{I}^{2}$ statistic. An $\mathrm{P}^{2}$ value of $>50 \%$ suggests substantial heterogeneity. We also extracted a $2 \times 2$ table to calculate $\mathrm{P}$ values for the comparisons among the results. For the purpose of statistical comparisons we chose bleomycin sclerotherapy as the reference group, since it is the sclerosing agent most commonly used in the USA. Meta-regression was not used in this study. Statistical analyses were performed using OpenMeta[Analyst] (http://www.cebm.brown.edu/openmeta/; Biostat, Englewood, NJ, USA).

\section{RESULTS}

\section{Literature search}

The initial literature search yielded 1,211 articles. On review of the abstracts and titles, we excluded 1,126 articles. Eighty- five articles were selected for full-text screening, of which 37 met inclusion criteria. ${ }^{1-37}$ The remaining 48 articles were excluded for reasons including 1) failure to separate outcomes by anatomic location (9 articles), 2) inclusion of lymphatic or venolymphatic malformations rather than pure VMs (14 articles), 3) use of confusing or unclear terminology making it difficult to ascertain whether lesions were VMs or hemangiomas (12 articles), and 4) mixture of VMs, AVMs and lymphatic malformations (13 articles). All studies included in the analysis had at least one or more outcome measure available for one or more of the patients groups analyzed. Fig. 1 shows the flow chart according to the PRISMA statement. ${ }^{37}$

These 37 studies included a total of 2,067 patients. The smallest study included 10 patients and the largest included 358 patients. Mean age was 24.9 years. There was a female predilection (1:1.2). The mean number of malformations per patient was 1.08 and they were all located in the head and/or neck region. The highest number of treated malformations per study was 358, while the least was 10 . The mean number of treatment sessions per patient was 2.4. The mean length of radiographic and clinical follow-up from the time of the first treatment was 16.61 months and 18.04 months respectively.

Most included studies used a single sclerosing agent for each vascular malformation, while the remainder used a combination of them. Five studies reported outcomes of bleomycin sclerotherapy, 10 studies reported outcomes of ethanol sclerotherapy, 7 studies reported outcomes of STS sclerotherapy, 4 studies reported outcomes of ethanolamine

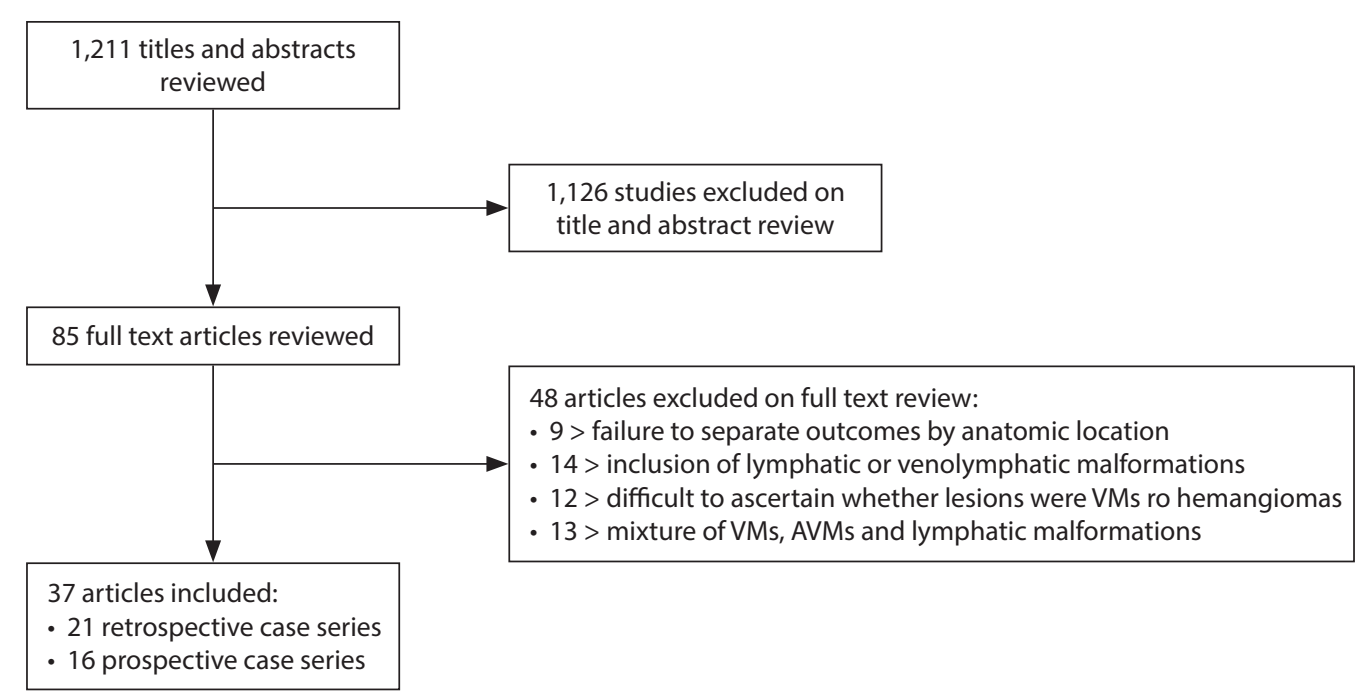

Fig. 1. PRISMA flow diagram. VMs, venous malformations; PRISMA, Preferred Reporting Items for Systematic Reviews and Meta-Analysis. 


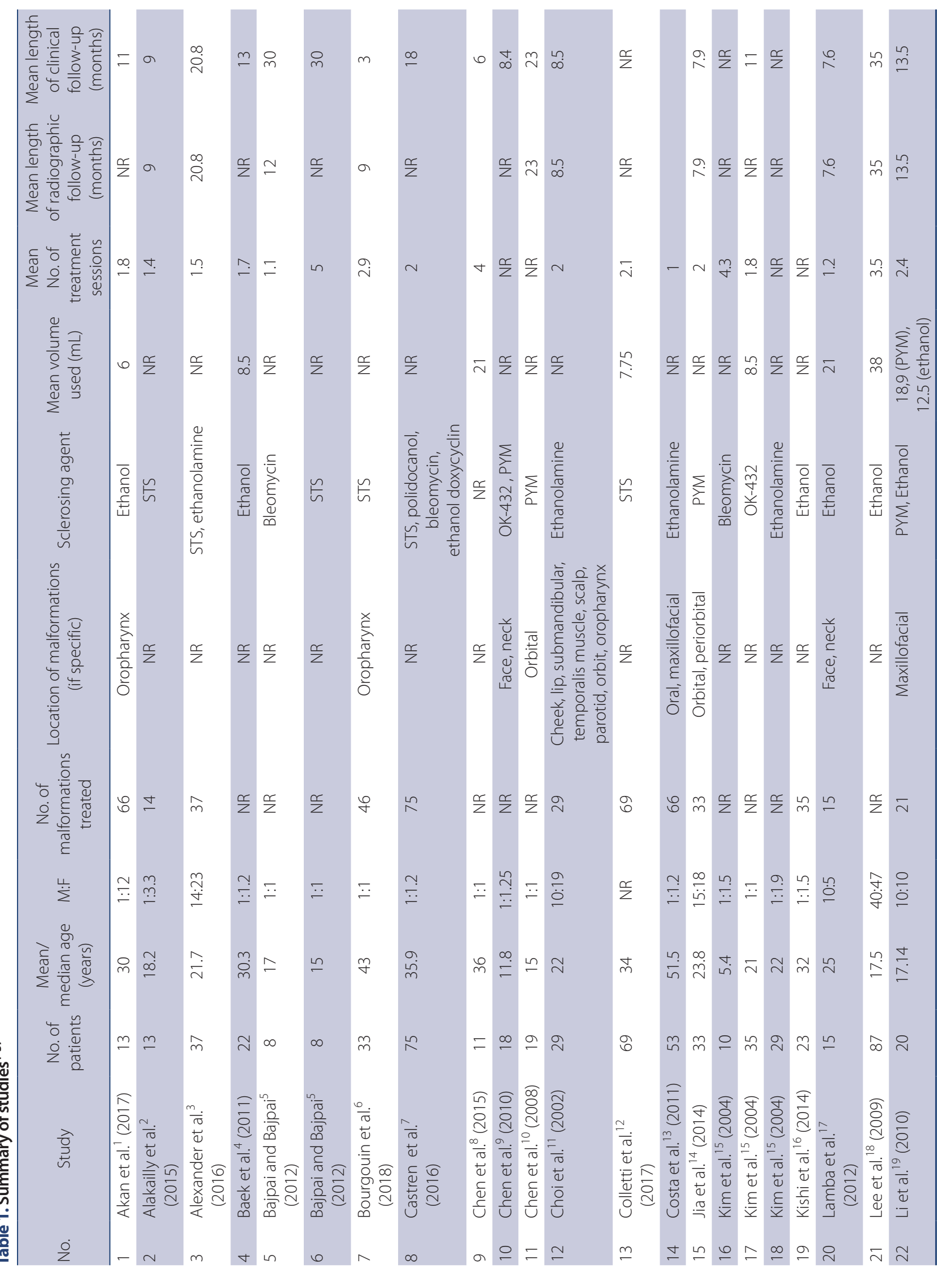




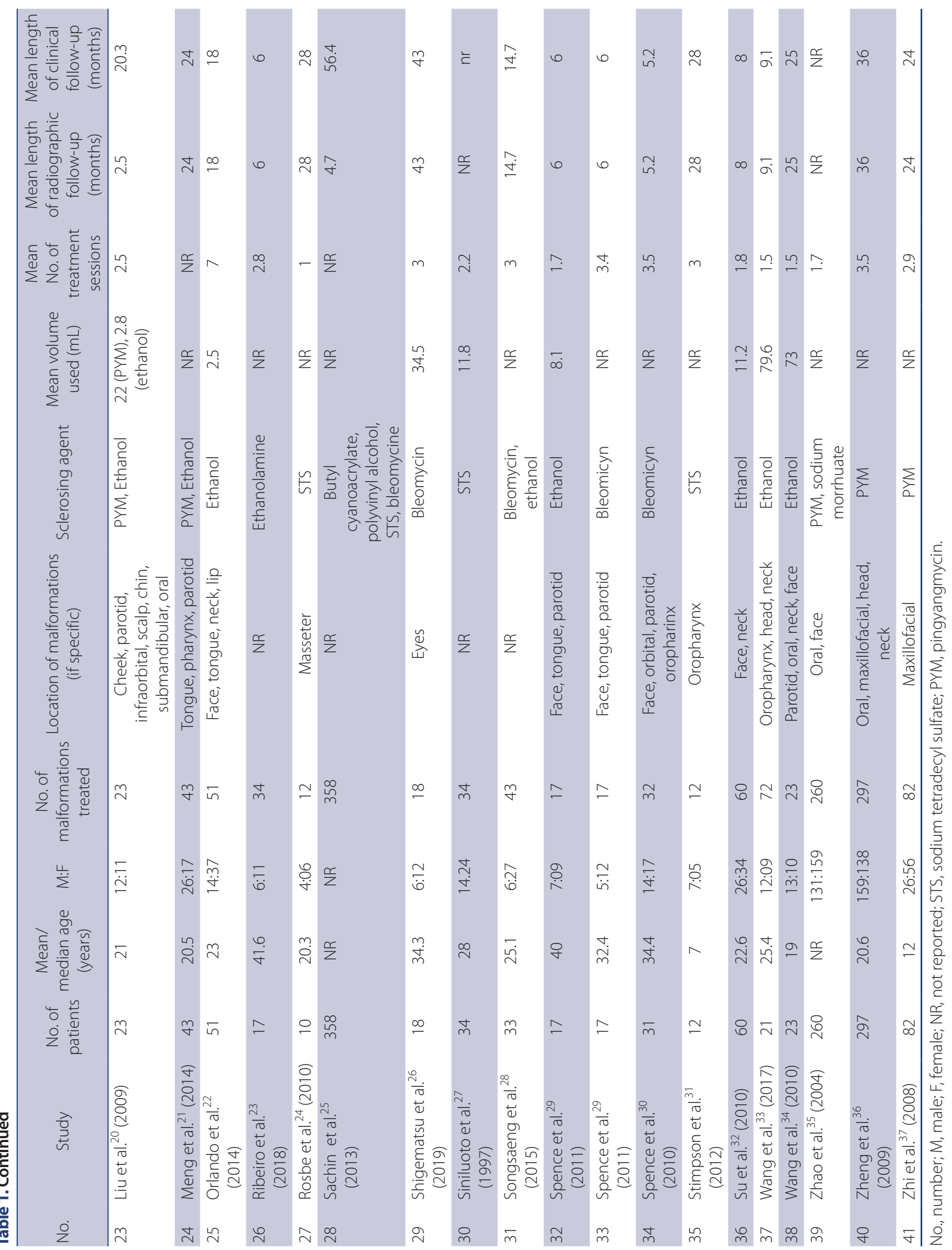


Study

Akan et al. ${ }^{1}$ (2017)

Baek et al. ${ }^{4}$ (2011)

Kishi et al. ${ }^{16}(2014)$

Lamba et al. ${ }^{17}$ (2012)

Lee et al. ${ }^{18}$ (2009)

Orlando et al. ${ }^{22}$ (2014)

Spence et al. ${ }^{29}(2011)$

Su et al. ${ }^{32}(2010)$

Wang et al. ${ }^{33}$ (2017)

Wang et al. ${ }^{34}(2010)$

Subgroup $2\left(I^{2}=9,673 \%, P=0.000\right)$

Alakailly et al. ${ }^{2}$ (2015)

Bajpai and Bajpai ${ }^{5}$ (2012) STS

Bourgouin et al. ${ }^{6}$ (2018)

Rosbe et al. ${ }^{24}(2010)$

Sachin et al. ${ }^{25}(2013)$

Siniluoto et al. ${ }^{27}$ (1997)

Stimpson et al. ${ }^{31}$ (2012)

Subgroup $3\left(\mathrm{I}^{2}=8,891 \%, \mathrm{P}=0.000\right)$

Alexander et al. ${ }^{3}$ (2016)

Castren et al. ${ }^{7}$ (2016)

Chen et al. ${ }^{8}(2015)$

Chen et al. ${ }^{9}(2010)$

Li et al. ${ }^{19}$ (2010)

Liu et al. ${ }^{20}$ (2009)

Meng et al. ${ }^{21}(2014)$

Songsaeng et al. ${ }^{28}$ (2015)

Zhao et al..$^{35}$ (2004)

Subgroup $5\left(\mathrm{I}^{2}=9,627 \%, \mathrm{P}=0.000\right)$

Bajpai and Bajpai ${ }^{5}$ (2012)

Shigematsu et al. ${ }^{26}(2019)$

Spence et al. ${ }^{29}$ (2011) bleo

Spence et al. ${ }^{30}(2010)$

Subgroup $1\left(I^{2}=9,446 \%, P=0.000\right)$

Chen et al. ${ }^{10}$ (2008)

Jia et al. ${ }^{14}$ (2014)

Zhi et al. ${ }^{37}$ (2008)

Zheng et al. ${ }^{36}(2009)$

Subgroup $7\left(I^{2}=8,771 \%, P=0.000\right)$

Choi et al. ${ }^{11}$ (2002)

Costa et al. ${ }^{13}(2011)$

Kim et al. ${ }^{15}$ (2004)

Ribeiro et al. ${ }^{23}$ (2018)

Subgroup $6\left(I^{2}=9,175 \%, P=0.000\right)$

Overall $\left(I^{2}=9,501 \%, P=0.000\right)$
Estimate $(95 \% \mathrm{Cl})$

$0.538(0.267,0.809)$

$0.409(0.204,0.615)$

$0.979(0.922,1.036)$

$0.286(0.049,0.522)$

$0.324(0.215,0.433)$

$0.275(0.152,0.397)$

$0.972(0.896,1.048)$

$0.933(0.870,0.996)$

$0.333(0.132,0.535)$

$0.739(0.560,0.919)$

$0.588(0.398,0.779)$

$0.308(0.057,0.559)$

$0.625(0.290,0.960)$

$0.364(0.200,0.528)$

$0.700(0.416,0.984)$

$0.824(0.778,0.869)$

$0.676(0.519,0.834)$

$0.333(0.067,0.600)$

$0.555(0.361,0.749)$

$0.981(0.928,1.034)$

$0.667(0.560,0.773)$

$0.364(0.079,0.648)$

$0.889(0.744,1.034)$

$0.650(0.441,0.859)$

$0.391(0.192,0.591)$

$0.163(0.052,0.273)$

$0.576(0.407,0.744)$

$0.712(0.656,0.767)$

$0.607(0.424,0.790)$

$0.875(0.646,1.104)$

$0.444(0.215,0.674)$

$0.969(0.883,1.054)$

$0.344(0.179,0.508)$

$0.661(0.313,1.009)$

$0.975(0.907,1.043)$

$0.758(0.611,0.904)$

$0.817(0.733,0.901)$

$0.749(0.685,0.812)$

$0.829(0.711,0.947)$

$0.483(0.301,0.665)$

$0.981(0.945,1.018)$

$0.759(0.603,0.914)$

$0.853(0.734,0.972)$

$0.783(0.589,0.976)$

$0.647(0.574,0.720)$

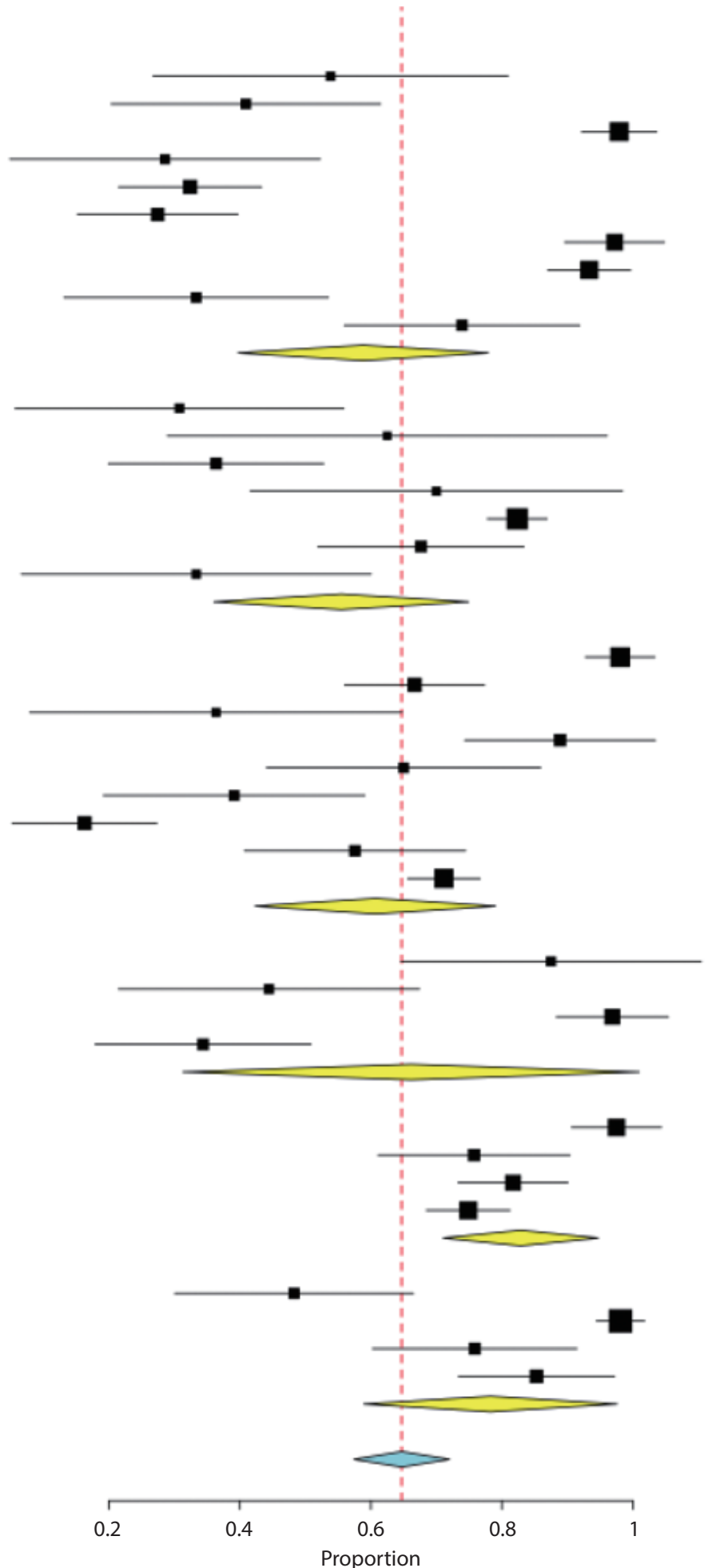

Proportion

Fig. 2. Forest plot: complete cure rates. Subgroup 1, bleomycin; subgroup 2, ethanol; subgroup 3, sotradecol; subgroup 5, mixed/other; subgroup 6 , ethanolamine; subgroup 7, pingyangmycin. Cl, confidence interval. 
sclerotherapy, and 4 studies reported outcomes of pingamycin sclerotherapy. In 11 studies either multiple agents were used and we could not separate outcomes by agent or other sclerosing agent including OK-432 were used. A summary of included studies is provided in Table 1.

Study

Akan et al. ${ }^{1}$ (2017)

Baek et al. ${ }^{4}(2011)$

Lamba et al. ${ }^{17}$ (2012)

Lee et al. ${ }^{18}$ (2009)

Orlando et al. ${ }^{22}$ (2014)

Spence et al. ${ }^{29}$ (2011)

Su et al. ${ }^{32}$ (2010)

Wang et al. ${ }^{33}$ (2017)

Wang et al. ${ }^{34}(2010)$

Subgroup $2\left(1^{2}=0 \%, P=0.981\right)$

Alakailly et al. ${ }^{2}$ (2015)

Bajpai and Bajpai ${ }^{5}$ (2012) STS

Bourgouin et al. ${ }^{6}(2018)$

Colletti et al. ${ }^{12}$ (2017)

Sachin et al. ${ }^{25}(2013)$

Siniluoto et al. ${ }^{27}$ (1997)

Stimpson et al. ${ }^{31}(2012)$

Subgroup $3\left(I^{2}=0 \%, P=0.831\right)$

Bajpai and Bajpai ${ }^{5}$ (2012)

Shigematsu et al. ${ }^{26}$ (2019)

Spence et al. ${ }^{29}$ (2011) bleo

Spence et al. ${ }^{30}(2010)$

Subgroup $1\left(I^{2}=0 \%, P=0.949\right)$

Castren et al. ${ }^{7}$ (2016)

Chen et al. ${ }^{8}$ (2015)

Chen et al. ${ }^{9}(2010)$

Li et al. ${ }^{19}(2010)$

Liu et al. ${ }^{20}$ (2009)

Meng et al. ${ }^{21}$ (2014)

Zhao et al. ${ }^{35}$ (2004)

Subgroup $5\left(I^{2}=0 \%, P=0.850\right)$

Chen et al. ${ }^{10}$ (2008)

Jia et al. ${ }^{14}$ (2014)

Zhi et al. ${ }^{37}$ (2008)

Subgroup $7\left(I^{2}=0 \%, P=0.820\right)$

Choi et al. ${ }^{11}$ (2002)

Kim et al. ${ }^{15}$ (2004)

Ribeiro et al. ${ }^{23}(2018)$

Subgroup $6\left(I^{2}=0 \%, P=0.966\right)$

Overall $\left(I^{2}=0 \%, P=1.000\right)$

\begin{tabular}{|c|c|}
\hline Estimate $(95 \% \mathrm{Cl})$ & EV/TRT \\
\hline $0.036(-0.061,0.133)$ & $0 / 13$ \\
\hline $0.022(-0.038,0.081)$ & $0 / 22$ \\
\hline $0.033(-0.058,0.124)$ & $0 / 14$ \\
\hline $0.006(-0.010,0.021)$ & $0 / 87$ \\
\hline $0.010(-0.017,0.036)$ & $0 / 51$ \\
\hline $0.059(-0.053,0.171)$ & $1 / 17$ \\
\hline $0.008(-0.014,0.031)$ & $0 / 60$ \\
\hline $0.023(-0.040,0.085)$ & $0 / 21$ \\
\hline $0.021(-0.036,0.078)$ & $0 / 23$ \\
\hline $0.010(-0.001,0.021)$ & $1 / 308$ \\
\hline $0.036(-0.061,0.133)$ & $0 / 13$ \\
\hline $0.056(-0.094,0.205)$ & $0 / 8$ \\
\hline $0.030(-0.028,0.089)$ & $1 / 33$ \\
\hline $0.007(-0.013,0.027)$ & $0 / 69$ \\
\hline $0.004(-0.004,0.011)$ & $1 / 272$ \\
\hline $0.029(-0.027,0.086)$ & $1 / 34$ \\
\hline $0.038(-0.066,0.143)$ & $0 / 12$ \\
\hline $0.005(-0.001,0.012)$ & $3 / 441$ \\
\hline $0.056(-0.094,0.205)$ & $0 / 8$ \\
\hline $0.026(-0.046,0.098)$ & $0 / 18$ \\
\hline $0.031(-0.054,0.117)$ & $0 / 15$ \\
\hline $0.015(-0.027,0.057)$ & $0 / 32$ \\
\hline $0.022(-0.011,0.054)$ & $0 / 73$ \\
\hline $0.040(-0.004,0.084)$ & $3 / 75$ \\
\hline $0.042(-0.071,0.155)$ & $0 / 11$ \\
\hline $0.026(-0.046,0.098)$ & $0 / 18$ \\
\hline $0.024(-0.014,0.089)$ & $0 / 20$ \\
\hline $0.021(-0.036,0.078)$ & $0 / 23$ \\
\hline $0.011(-0.020,0.043)$ & $0 / 43$ \\
\hline $0.006(-0.011,0.023)$ & $0 / 82$ \\
\hline $0.012(-0.001,0.025)$ & $3 / 272$ \\
\hline $0.025(-0.043,0.093)$ & $0 / 19$ \\
\hline $0.015(-0.026,0.055)$ & $0 / 33$ \\
\hline $0.006(-0.011,0.023)$ & $0 / 82$ \\
\hline $0.008(-0.007,0.023)$ & $0 / 134$ \\
\hline $0.017(-0.029,0.062)$ & $0 / 29$ \\
\hline $0.017(-0.029,0.062)$ & $0 / 29$ \\
\hline $0.028(-0.048,0.104)$ & $0 / 17$ \\
\hline $0.018(-0.011,0.048)$ & $0 / 75$ \\
\hline $0.008(0.003,0.013)$ & $1 / 1,303$ \\
\hline
\end{tabular}

\section{Efficacy outcomes}

Overall complete cure rates were reported in 1,736 patients. The overall rate of complete cure following percutaneous sclerotherapy with any agent was $64.7 \%(95 \% \mathrm{Cl}, 57.4-72.0 \%)$. STS had the lowest complete cure rate at $55.5 \%(95 \% \mathrm{Cl}$,

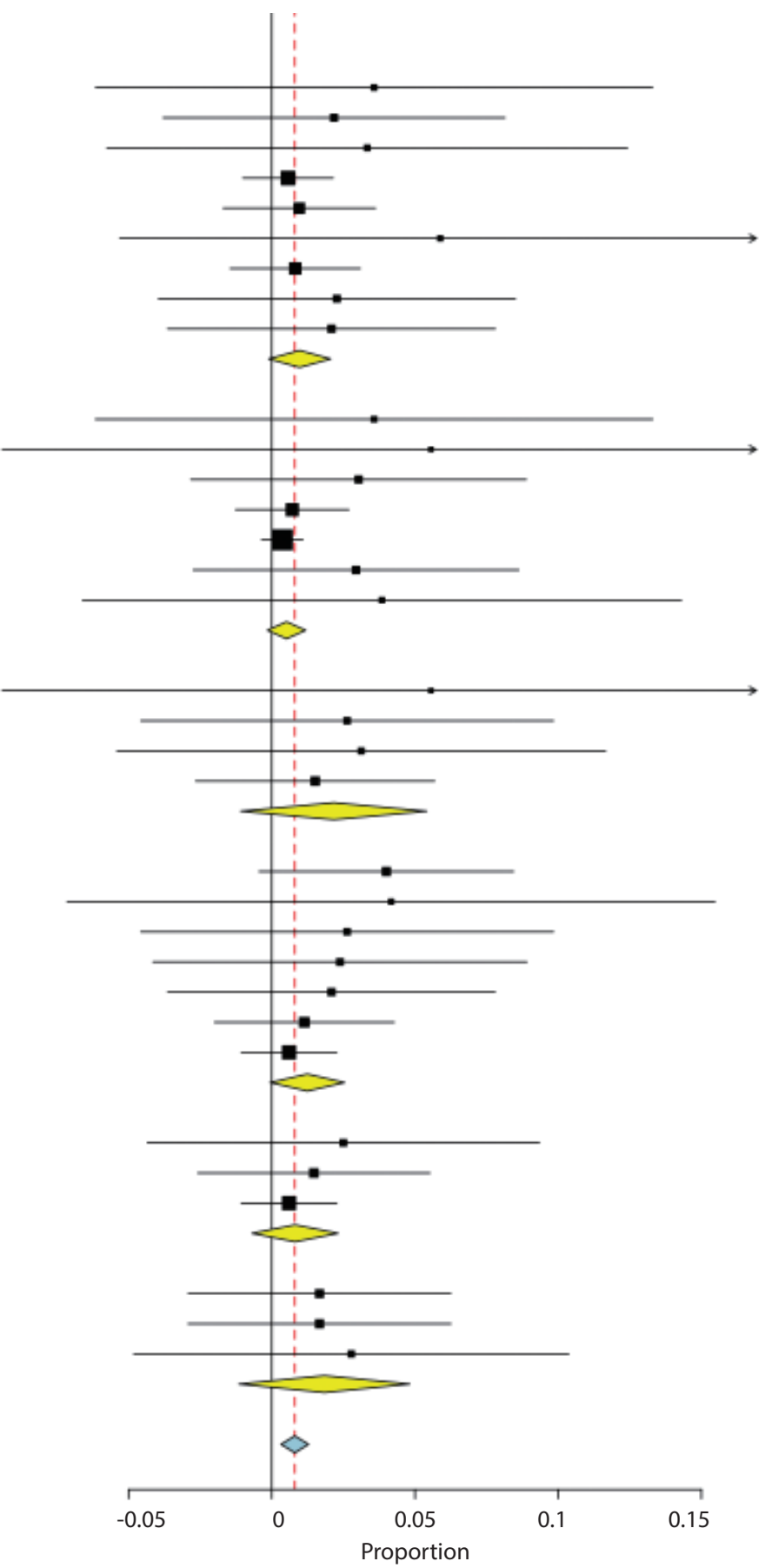

Fig. 3. Forest plot: permanent morbidity and mortality rates. Subgroup 1, bleomycin; subgroup 2, ethanol; subgroup 3, sotradecol; subgroup 5, mixed/other; subgroup 6, ethanolamine; subgroup 7, pingyangmycin. Cl, confidence intervall; EV, number of events; TRT, number of treated patients. 
36.1-74.9\%) while pingyangmycin had the highest cure rate at $82.9 \%$ (95\% Cl, 71.1-94.7\%). Fig. 2 shows the complete cure rates forest plot. Overall partial cure rates were reported in 1,703 patients. The overall rate of partial cure following percutaneous sclerotherapy with any agent was $28.0 \%$ (95\% Cl, 22.1-34.0\%). Partial cure rates ranged from $16.2 \%$ (95\% Cl,
4.7-27.6\%) for pingyangmycin to $35.3 \%$ (95\% Cl, 19.1-51.5\%) with ethanol. The overall rate of no benefit following percutaneous sclerotherapy was reported in 1,736 patients and was $4.5 \%(95 \% \mathrm{Cl}, 3.0-6.1 \%)$. STS sclerotherapy had the highest rate of no benefit (14.9\%; 95\% Cl, 4.3-25.4\%) while pingyangmycin had the lowest rate of no benefit $(0.6 \%$;
Study

Akan et al. ${ }^{1}$ (2017)

Baek et al. ${ }^{4}(2011)$

Kishi et al. ${ }^{16}$ (2014)

Lamba et al. ${ }^{17}$ (2012)

Lee et al. ${ }^{18}(2009)$

Orlando et al. ${ }^{22}$ (2014)

Spence et al. ${ }^{29}(2011)$

Su et al. ${ }^{32}$ (2010)

Wang et al. ${ }^{33}(2017)$

Wang et al. ${ }^{34}(2010)$

Subgroup $2\left(\mathrm{I}^{2}=0 \%, \mathrm{P}=0.998\right)$

Alakailly et al. ${ }^{2}$ (2015)

Bajpai and Bajpai ${ }^{5}$ (2012) STS

Bourgouin et al. ${ }^{6}$ (2018)

Colletti et al. ${ }^{12}$ (2017)

Sachin et al. ${ }^{25}$ (2013)

Siniluoto et al. ${ }^{27}$ (1997)

Stimpson et al. ${ }^{31}(2012)$

Subgroup $3\left(1^{2}=0 \%, P=0.011\right)$

Bajpai and Bajpai ${ }^{5}$ (2012)

Shigematsu et al. ${ }^{26}$ (2019)

Spence et al. ${ }^{29}$ (2011) bleo

Spence et al. ${ }^{30}(2010)$

Subgroup $1\left(\mathrm{I}^{2}=0 \%, \mathrm{P}=0.949\right)$

Chen et al. ${ }^{8}$ (2015)

Chen et al..$^{9}$ (2010)

Li et al..$^{19}$ (2010)

Liu et al. ${ }^{20}$ (2009)

Meng et al. ${ }^{21}$ (2014)

Zhao et al. ${ }^{35}$ (2004)

Subgroup $5\left(I^{2}=0 \%, P=0.960\right)$

Chen et al. ${ }^{10}$ (2008)

Jia et al. ${ }^{14}$ (2014)

Zhi et al. ${ }^{37}$ (2008)

Subgroup $7\left(I^{2}=0 \%, P=0.820\right)$

Choi et al. ${ }^{11}$ (2002)

Ribeiro et al. ${ }^{23}$ (2018)

Subgroup $6\left(I^{2}=0 \%, P=0.806\right)$

Overall $\left(I^{2}=0 \%, P=0.771\right)$

\begin{tabular}{|c|c|}
\hline Estimate $(95 \% \mathrm{Cl})$ & $\mathrm{EV} / \mathrm{TRT}$ \\
\hline $0.036(-0.061,0.133)$ & $0 / 13$ \\
\hline $0.022(-0.038,0.081)$ & $0 / 22$ \\
\hline $0.021(-0.036,0.078)$ & $0 / 23$ \\
\hline $0.033(-0.058,0.124)$ & $0 / 14$ \\
\hline $0.023(-0.009,0.054)$ & $2 / 87$ \\
\hline $0.010(-0.017,0.036)$ & $0 / 51$ \\
\hline $0.028(-0.048,0.104)$ & $0 / 17$ \\
\hline $0.008(-0.014,0.031)$ & $0 / 60$ \\
\hline $0.023(-0.040,0.085)$ & $0 / 21$ \\
\hline $0.021(-0.036,0.078)$ & $0 / 23$ \\
\hline $0.015(0.002,0.028)$ & $2 / 331$ \\
\hline $0.036(-0.061,0.133)$ & $0 / 13$ \\
\hline $0.056(-0.094,0.205)$ & $0 / 8$ \\
\hline $0.303(0.146,0.460)$ & $10 / 33$ \\
\hline $0.014(-0.014,0.043)$ & $1 / 69$ \\
\hline $0.002(-0.003,0.007)$ & $0 / 272$ \\
\hline $0.014(-0.025,0.054)$ & $0 / 34$ \\
\hline $0.038(-0.066,0.143)$ & $0 / 12$ \\
\hline $0.024(-0.005,0.053)$ & $11 / 441$ \\
\hline $0.056(-0.094,0.205)$ & $0 / 8$ \\
\hline $0.026(-0.046,0.098)$ & $0 / 18$ \\
\hline $0.031(-0.054,0.117)$ & $0 / 15$ \\
\hline $0.015(-0.027,0.057)$ & $0 / 32$ \\
\hline $0.022(-0.011,0.054)$ & $0 / 73$ \\
\hline $0.042(-0.071,0.155)$ & $0 / 11$ \\
\hline $0.026(-0.046,0.098)$ & $0 / 18$ \\
\hline $0.024(-0.041,0.089)$ & $0 / 20$ \\
\hline $0.021(-0.036,0.078)$ & $0 / 23$ \\
\hline $0.011(-0.020,0.043)$ & $0 / 43$ \\
\hline $0.006(-0.011,0.023)$ & $0 / 82$ \\
\hline $0.010(-0.004,0.023)$ & 0/197 \\
\hline $0.025(-0.043,0.093)$ & $0 / 19$ \\
\hline $0.015(-0.026,0.055)$ & $0 / 33$ \\
\hline $0.006(-0.011,0.023)$ & $0 / 82$ \\
\hline $0.008(-0.007,0.023)$ & $0 / 134$ \\
\hline $0.017(-0.029,0.062)$ & $0 / 29$ \\
\hline $0.028(-0.048,0.104)$ & $0 / 17$ \\
\hline $0.020(-0.020,0.059)$ & $0 / 46$ \\
\hline $0.006(0.002,0.010)$ & $13 / 1,222$ \\
\hline
\end{tabular}

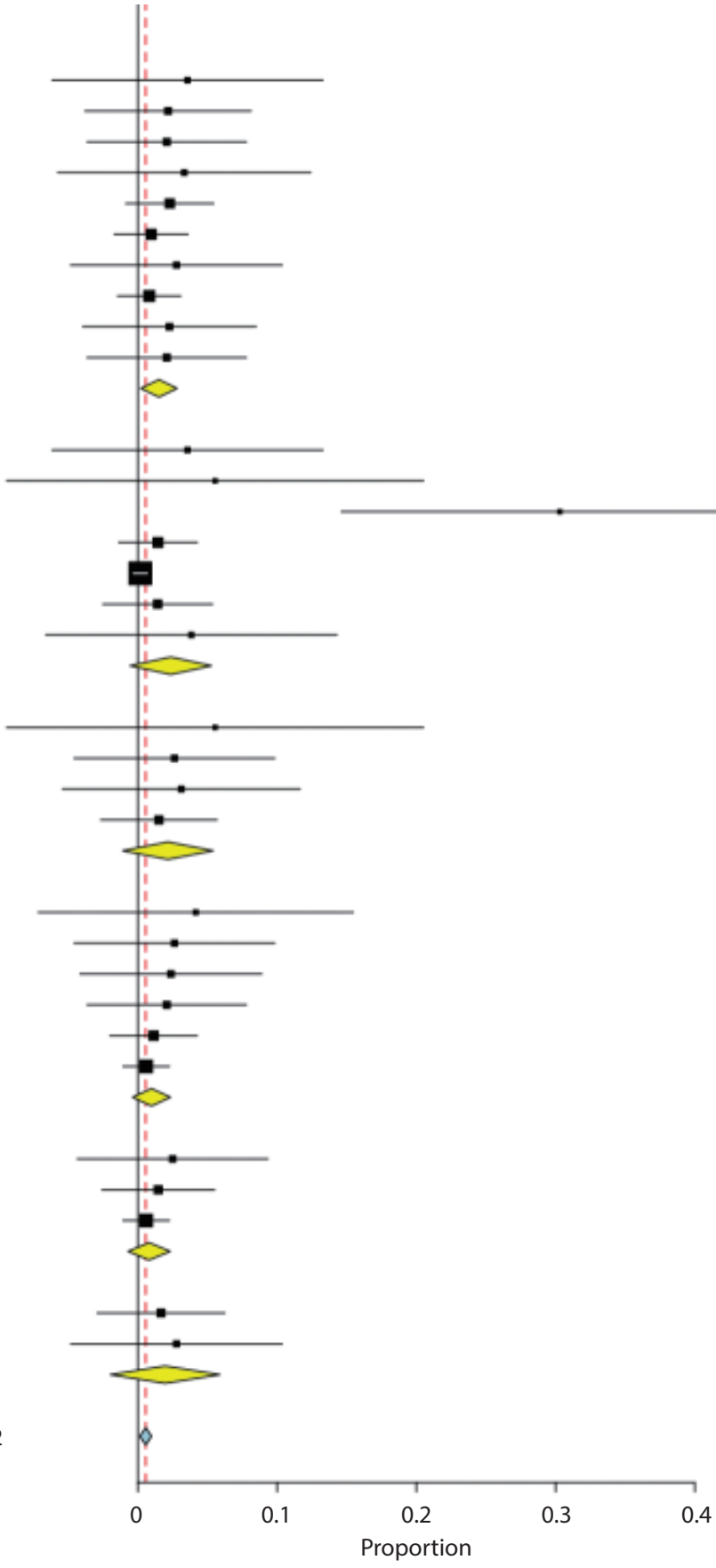

Fig. 4. Forest plot: pulmonary complication rates. Subgroup 1, bleomycin; subgroup 2, ethanol; subgroup 3, sotradecol; subgroup 5, mixed/other; subgroup 6, ethanolamine; subgroup 7, pingyangmycin. Cl, confidence interval; EV, number of events; TRT, number of treated patients. 
95\% Cl, 0.0-1.6\%). Patient satisfaction was reported in 315 patients. Overall patient satisfaction rates were $91.0 \%$ (95\% Cl, 86.1-95.9\%). Patient satisfaction rates ranged from $72.8 \%$ (95\% Cl, 63.6-81.9\%) with STS to $96.0 \%$ (95\% Cl, 92.5-99.6\%) with ethanol. Improvement in QoL was reported in 243 patients. Overall QoL improvement was 78.9\% (95\% Cl, 67.0-90.8\%) ranging from $46.7 \%(95 \% \mathrm{Cl}, 22.4-71.0 \%)$ for STS to $98.1 \%$ (95\% Cl, 94.5-100\%) with ethanolamine.

\section{Safety outcomes}

Overall permanent morbidity and mortality rates were reported in 1,303 patients. The overall rate of permanent mor-
Study

Baek et al. ${ }^{4}$ (2011)

Lamba et al. ${ }^{17}(2012)$

Lee et al. ${ }^{18}$ (2009)

Orlando et al. ${ }^{22}$ (2014)

Spence et al. ${ }^{29}(2011)$

Su et al. ${ }^{32}(2010)$

Wang et al. ${ }^{33}(2017)$

Wang et al. ${ }^{34}(2010)$

Subgroup $2\left(I^{2}=255 \%, P=0.413\right)$

Alakailly et al. ${ }^{2}$ (2015)

Bajpai and Bajpai ${ }^{5}$ (2012) STS

Bourgouin et al. ${ }^{6}$ (2018)

Colletti et al. ${ }^{12}$ (2017)

Sachin et al. ${ }^{25}$ (2013)

Siniluoto et al. ${ }^{27}$ (1997)

Stimpson et al. ${ }^{31}$ (2012)

Subgroup $3\left(I^{2}=0 \%, P=0.603\right)$

Bajpai and Bajpai $^{5}$ (2012)

Shigematsu et al. ${ }^{26}$ (2019)

Spence et al. ${ }^{29}$ (2011) bleo

Spence et al. ${ }^{30}(2010)$

Subgroup $1\left(I^{2}=0 \%, P=0.853\right)$

Chen et al. ${ }^{8}$ (2015)

Chen et al. ${ }^{9}(2010)$

Li et al. ${ }^{19}$ (2010)

Liu et al. ${ }^{20}$ (2009)

Meng et al. ${ }^{21}$ (2014)

Zhao et al. ${ }^{35}$ (2004)

Subgroup $5\left(I^{2}=0 \%, P=0.506\right)$

Chen et al. ${ }^{10}$ (2008)

Jia et al. ${ }^{14}(2014)$

Zhi et al. ${ }^{37}$ (2008)

Ribeiro et al. ${ }^{23}(2018)$

Subgroup $6\left(I^{2}=0 \%, P=0.806\right)$

Overall $\left(I^{2}=0 \%, P=0.904\right)$
Akan et al. ${ }^{1}$ (2017)

Castren et al. ${ }^{7}$ (2016)

Subgroup $7\left(I^{2}=0 \%, P=0.820\right)$

Choi et al. ${ }^{11}$ (2002)

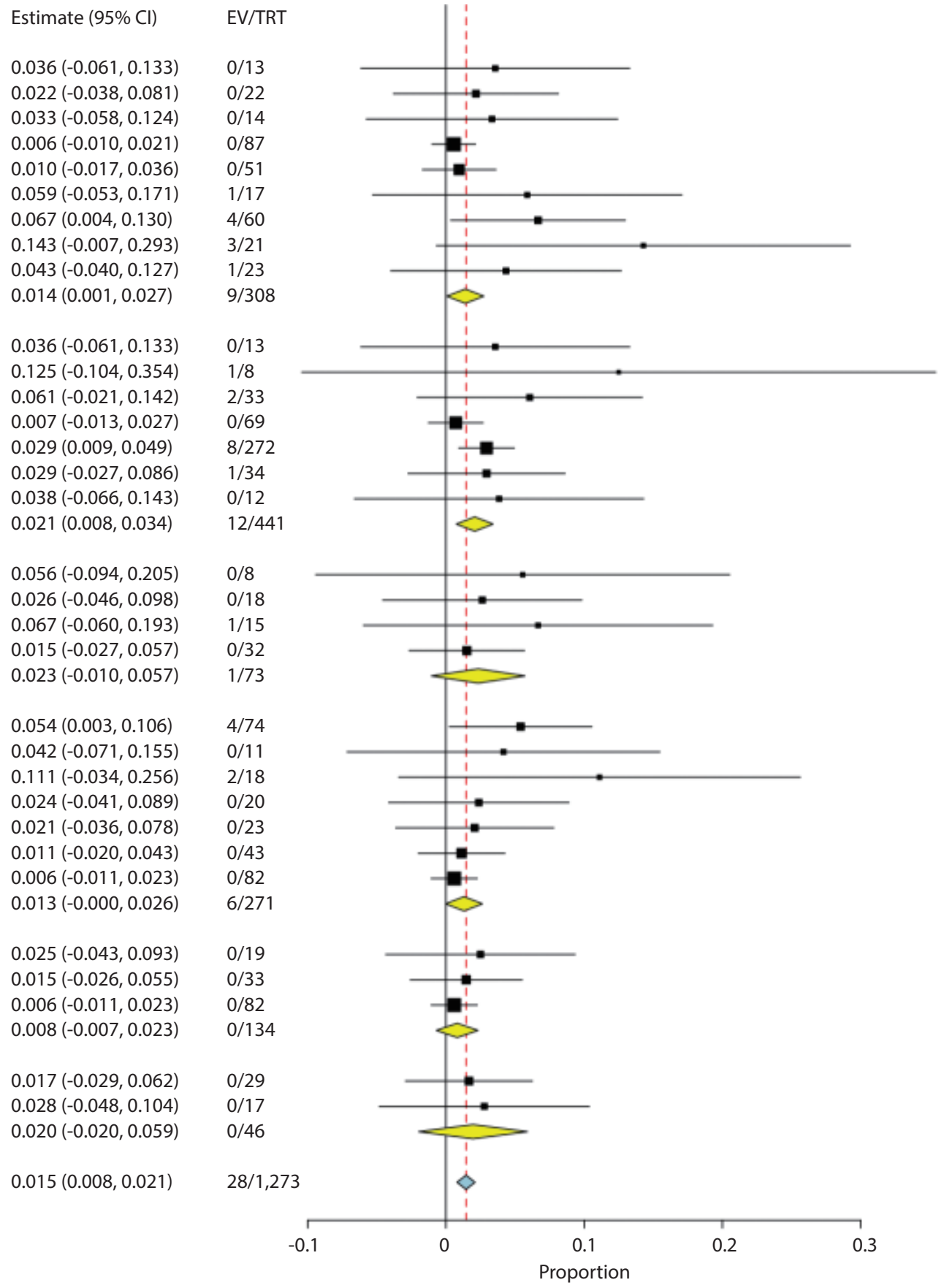

Fig. 5. Forest plot: skin necrosis and scarring rates. Subgroup 1, bleomycin; subgroup 2, ethanol; subgroup 3, sotradecol; subgroup 5, mixed/other; subgroup 6, ethanolamine; subgroup 7, pingyangmycin. Cl, confidence interval; EV, number of events; TRT, number of treated patients. 
bidity and mortality was $0.8 \%(95 \% \mathrm{Cl}, 0.3-1.3 \%)$. The lowest rate was reported with STS, and pingamycin $(0.5 \%, 0.8 \%)$ and the highest rate reported was with bleomycin $(2.2 \% ; 95 \% \mathrm{Cl}$, 0.0-5.4\%). Fig. 3 shows the permanent morbidity and mortality rates forest plot. Local temporary complication rates were reported in 1,312 patients. The overall rate of local temporary complications was $41.8 \%$ (95\% Cl, 27.0-56.5\%). Local temporary complications were highest with ethanolamine (51.0\%; 95\% Cl, 24.7-26.6\%) and were lowest with bleomycin (27.0\%; 95\% Cl, 5.4-59.4\%). Pulmonary complication rates were reported in 1,222 patients. The overall rate was $0.6 \%$ (95\% Cl, 0.2-1.0\%). Pulmonary complication rate was lowest for pingamycin $(0.8 \% ; 95 \% \mathrm{Cl}, 0.0-2.3 \%)$, and was highest for STS (2.4\%; 95\% Cl, 0.5-5.3\%). Fig. 4 shows the pulmonary complication rates forest plot. Skin necrosis and scarring rates were reported in 1,273 patients and the overall rate was $1.5 \%$ (95\% Cl, 0.8-2.1\%). Rates were highest with bleomycin (2.3\%; $95 \% \mathrm{Cl}, 1.0-5.7 \%)$ and lowest with pingamycin (0.8\%; $95 \% \mathrm{Cl}, 0.0-2.3 \%)$. Fig. 5 shows the skin necrosis and scarring rates forest plot. The efficacy and safety outcomes are summarized in Tables 2 and 3.

\section{Study heterogeneity}

$I^{2}$ values were $>50 \%$ indicating substantial heterogeneity for the following outcomes: lack of benefit, improvement in QoL, local temporary complication, overall cure, partial cure, patient satisfaction and systemic side effects. $I^{2}$ values were $<50 \%$ indicating lack of substantial heterogeneity for the following outcomes: permanent morbidity and mortality, pulmonary complications and skin necrosis/scarring.

Table 2. Summary of overall outcomes

\begin{tabular}{lc}
\hline & Overall (\%) (95\% Cl) \\
\hline Complete cure & $64.7(57.4-72.0)$ \\
\hline Partial cure & $28.0(22.1-34.0)$ \\
No benefit & $4.5(3.0-6.1)$ \\
Improvement in QoL & $78.9(67.0-90.8)$ \\
Patient satisfaction & $91.0(86.1-95.9)$ \\
Pulmonary complication & $0.6(0.2-1.0)$ \\
Skin necrosis/scar & $1.5(0.8-2.1)$ \\
Any permanent morbidity/mortality & $0.8(0.3-1.3)$ \\
Local temporary complication & $41.8(27.0-56.5)$ \\
\hline
\end{tabular}

QoL, quality of life; $\mathrm{Cl}$, confidence interval.

\section{DISCUSSION}

Our systematic review and meta-analysis of percutaneous image guided sclerotherapy for treatment of VMs of the head face and neck found that the overall efficacy rate of sclerotherapy was high with complete and partial cure rates of over $90 \%$. Over $90 \%$ of patients reported satisfaction with the results of their sclerotherapy treatments and over $70 \%$ reported improvements in QoL. Temporary local complication rates were high, however rates of permanent morbidity and mortality as well as permanent scarring were low. When performing subgroup analyses by agent, we found that agents such as ethanol, pingamycin and ethanolamine were generally associated with the highest rates of cure and patient satisfaction, however these agents were also associated with higher rates of local temporary complications. These findings are important as they provide important information regarding the risks and benefits of percutaneous sclerotherapy for VMs of the head, face and neck.

The efficacy of sclerotherapy for VMs is dependent on both the angioarchitecture of the venous malformation, the sclerosing agent used and the dwell time of the sclerosant within the venous malformation. ${ }^{42}$ Angioarchitecture can be easily assessed on contrast venography following percutaneous injection of the lesion. According to Gemmette et al, the 4 morphological subtypes of VMs are as follows: type I, isolated malformation without discernible venous drainage; type II, lesions draining into normal veins; type III, lesions draining into dysplastic veins; type IV, lesion consists primarily of venous ectasia. ${ }^{42}$ Types I and II are thought to respond best to sclerotherapy. None of the studies included in our analysis examined outcomes by lesion angioarchitecture, however this undoubtedly is an important factor in determining outcomes.

A summary of the different sclerosing agents is provided in Table 4. Based on our study, it is clear that sclerosants such as ethanol, ethanolamine oleate and pingyangmycin are generally more effective at inducing a complete or partial cure of the VM. However, this comes at the cost of increased risk of permanent morbidity as well as local side effects. Medications such as ethanol, ethanolamine and pingyangmycin act by directly inducing endothelial injury and thrombosis of the VM. ${ }^{43}$ Meanwhile, less potent sclerosing agents such as STS and bleomycin work by inducing a nonspecific inflammatory reaction within the $\mathrm{VM}^{43}$ Adverse side effects of sclerosing agents also differ by agent and are summarized in Table 4 . 


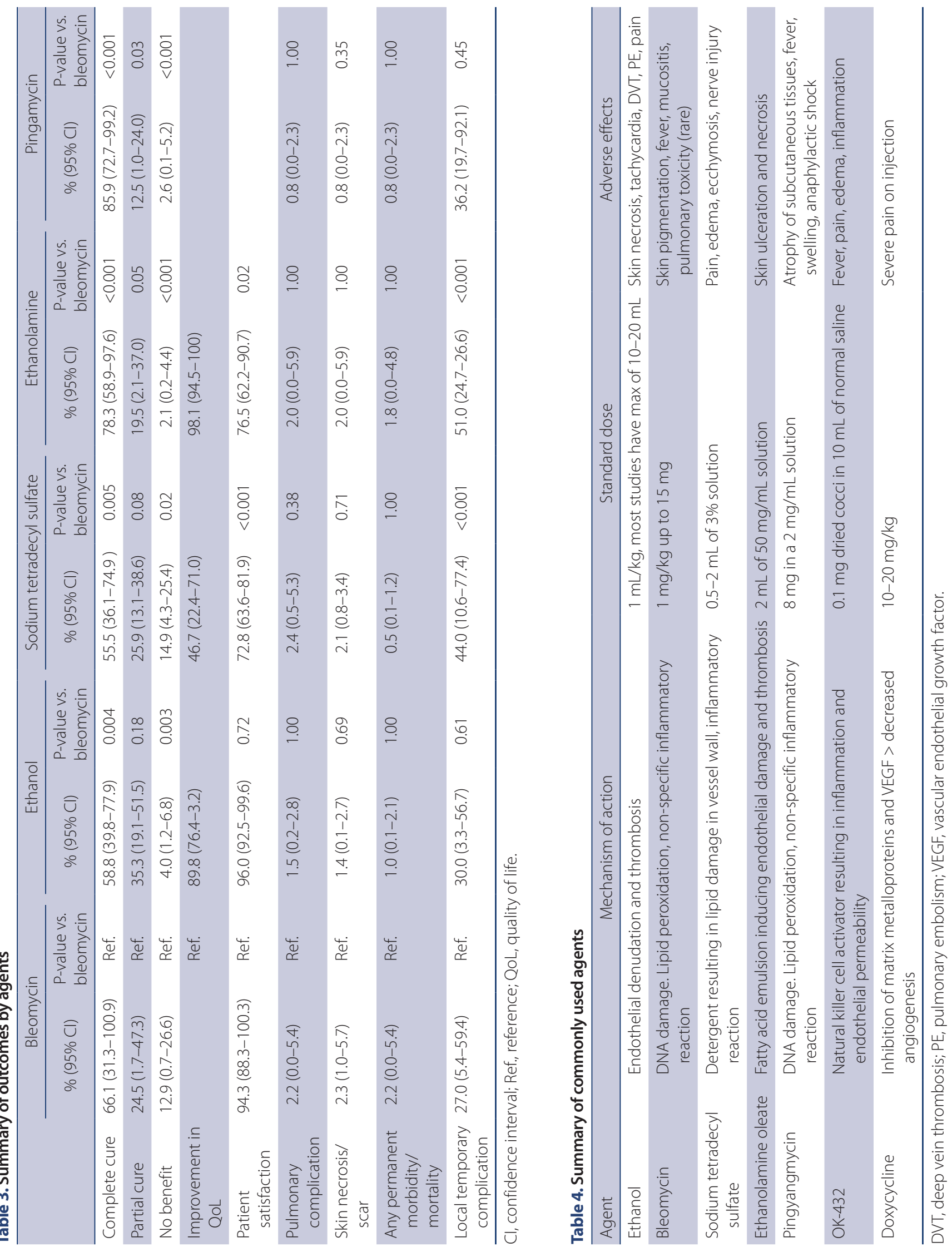


In brief, adverse side effects are generally most severe with ethanol and include nerve injury, skin necrosis, and cardiopulmonary collapse. ${ }^{43}$ Side effects are least severe with bleomycin and include mucositis and skin pigmentation.

It is important to point out that a variety of factors should go into the decision regarding which sclerotherapeutic agent to use. Choice of agent should be based on the depth of the venous malformation from the skin surface as well as the ability to limit non-target sclerotherapy and venous thrombosis. Alcohol is generally best used for deeper VMs due to the risk of skin necrosis from extravasation. ${ }^{4,28,29} \mathrm{Su}$ perficial VMs are likely best treated with milder agents such as bleomycin or STS. $5,26,28,29$

The results from our study compare favorably to other modalities of treatment including medical therapy, surgical resection and laser therapy. Conservative management techniques for VMs are centered around pain control, prevention of phlebitis or thrombosis and prevention of bleeding. ${ }^{43}$ Pain can generally be managed with anti-inflammatory medications and analgesics. Low dose aspirin can provide pain relief in patients with venous malformation thrombosis. ${ }^{24,31}$ Recently the mammalian target of rapamycin inhibitor sirolismus has been trialed for treatment of VMs and has been found to result in symptom improvement in approximately $80 \%$ of patients. ${ }^{44}$ Surgical excision of VMs is usually part of staged multimodal treatment of VMs. ${ }^{45}$ However, surgical excision alone is often considered when lesions are smaller and are not adjacent to vital structures. Surgical excision of large invasive VMs is often very challenging due to the presence of poorly defined tissue planes and proximity to important structures. In such cases, intralesional sclerotherapy can be considered as a useful adjunct to surgery as part of an interdisciplinary approach. ${ }^{46}$ Small case series have reported various levels of efficacy with surface and endovenous/interstitial laser therapy for treatment of VMs, most series report efficacy rates ranging from $70-90 \%$. Combined laser and sclerotherapy treatment has also been shown to be effective as part of an interdisciplinary approach. ${ }^{46}$

\section{Limitations}

Our study has limitations. This meta-analysis was based primarily off of single-center case series and thus has limitations inherent to single center retrospective studies. There was high risk of bias in 21 series. While we were able to perform subgroup analyses based on sclerotherpeutic agent used, we were unable to perform more granular analyses stratify- ing outcomes by lesion location. Follow-up in the included studies is limited. Many of our outcomes are subjective and volumetric analyses documenting lesion improvement were not available for a vast majority of the included studies. Nonetheless, our study provides helpful information for both patients and providers who are considering percutaneous sclerotherapy for treatment of VMs and provides guidance for future areas of investigation.

\section{CONCLUSIONS}

Our systematic review and meta-analysis of 37 studies and over 2,000 patients found that percutaneous sclerotherapy is a very safe and effective treatment modality for treatment of VMs of the head, neck and face. As expected, mild sclerosing agents such as bleomycin and STS were associated with lower efficacy rates but generally superior safety profiles than stronger sclerosing agents such as ethanol, ethanolamine and pingyangmycin. Further prospective studies are needed to validate our results and determine the optimal treatment strategies for patients with VMs of the head, face and neck.

\section{Fund}

None.

\section{Ethics Statement}

For this type of study formal consent is not required.

\section{Conflicts of Interest}

The authors have no conflicts to disclose.

\section{REFERENCES}

1. Akan H, Uzunkaya F, Soylu Al. Percutaneous ethanol sclerotherapy of venous malformations of the oral cavity and the oropharynx. Erciyes Med 2017:39:183-188

2. Alakailly X, Kummoona R, Quereshy FA, Baur DA, González AE. The use of sodium tetradecyl sulphate for the treatment of venous malformations of the head and neck. J Maxillofac Oral Surg 2015;14:332-338

3. Alexander MD, McTaggart RA, Choudhri OA, Pandit RP, Wu A, Ross M, et al. Quantitative volumetric analysis of head and neck venous and lymphatic malformations to assess response to percutaneous sclerotherapy. Acta Radio/ 2016;57:205-209 
4. Baek HJ, Hong JP, Choi JW, Suh DC. Direct percutaneous alcohol sclerotherapy for venous malformations of head and neck region without fluoroscopic guidance: technical consideration and outcome. Neurointervention 2011;6:84-88

5. Bajpai H, Bajpai S. Comparative analysis of intralesional sclerotherapy with sodium tetradecyl sulfate versus bleomycin in the management of low flow craniofacial soft tissue vascular lesions. J Maxillofac Oral Surg 2012;11:13-20

6. Bourgouin $P$, Thomas-Chaussé F, Gilbert $P$, Giroux MF, Périgny $S$, Guertin $L$, et al. Effectiveness and safety of sclerotherapy for treatment of low-flow vascular malformations of the oropharyngeal region. J Vasc Interv Radiol 2018;29:809-815

7. Castren E, Aronniemi J, Klockars T, Pekkola J, Lappalainen K, Vuola P, et al. Complications of sclerotherapy for 75 head and neck venous malformations. Eur Arch Otorhinolaryngol 2016;273:1027-1036

8. Chen AW, Liu YR, Li K, Zhang K, Wang T, Liu SH. Efficacy of sclerotherapy with radio-opaque foam guided by digital subtraction angiography for the treatment of complex venous malformations of the head and neck. Br J Oral Maxillofac Surg 2015;53:809-813

9. Chen WL, Huang ZQ, Zhang DM, Chai Q. Percutaneous sclerotherapy of massive venous malformations of the face and neck using fibrin glue combined with ok-432 and pingyangmycin. Head Neck 2010;32:467-472

10. Chen Y, Li Y, Zhu Q, Zeng Q, Zhao J, He X, et al. Fluoroscopic intralesional injection with pingyangmycin lipiodol emulsion for the treatment of orbital venous malformations. AJR Am J Roentgenol 2008;190:966-971

11. Choi YH, Han MH, O-Ki K, Cha SH, Chang KH. Craniofacial cavernous venous malformations: percutaneous sclerotherapy with use of ethanolamine oleate. J Vasc Interv Radiol 2002;13:475-482

12. Colletti G, Deganello A, Bardazzi A, Mattassi R, Dalmonte P, Gazzabin $L$, et al. Complications after treatment of head and neck venous malformations with sodium tetradecyl sulfate foam. J Craniofac Surg 2017;28:e388-e392

13. Costa JR, Torriani MA, Hosni ES, D'Avila OP, de Figueiredo PJ. Sclerotherapy for vascular malformations in the oral and maxillofacial region: treatment and follow-up of 66 lesions. J Oral Maxillofac Surg 2011;69:e88-e92

14. Jia R, Xu S, Huang X, Song X, Pan H, Zhang L, et al. Pingyangmycin as first-line treatment for low-flow orbital or periorbital venous malformations: evaluation of 33 consecutive patients. JAMA Ophthalmol 2014;132:942-948

15. Kim KH, Sung MW, Roh JL, Han MH. Sclerotherapy for congen- ital lesions in the head and neck. Otolaryngol Head Neck Surg 2004;131:307-316

16. Kishi K, Morita N, Terada T, Sato M. Dose-saving isolation procedure in percutaneous ethanol sclerotherapy for venous malformations. Phlebology 2014;29:276-286

17. Lamba S, Keshava SKN, Moses V, Surendrababu N, Gupta AK. Ethanol sclerotherapy for treatment of venous malformations of face and neck-a single centre experience. Eur J Plast Surg 2012;35:345-350

18. Lee $\mathrm{IH}$, Kim KH, Jeon P, Byun HS, Kim HJ, Kim ST, et al. Ethanol sclerotherapy for the management of craniofacial venous malformations: the interim results. Korean J Radio/ 2009;10:269-276

19. Li J, Chen J, Zheng G, Liao G, Fu Z, Li J, et al. Digital subtraction angiography-guided percutaneous sclerotherapy of venous malformations with pingyangmycin and/or absolute ethanol in the maxillofacial region. J Oral Maxillofac Surg 2010;68:22582266

20. Liu Y, Liu D, Wang Y, Zhang W, Zhao F. Clinical study of sclerotherapy of maxillofacial venous malformation using absolute ethanol and pingyangmycin. J Oral Maxillofac Surg 2009;67:98104

21. Meng J, Zhuang QW, Gu QP, Zhang J, Li ZP, Si YM. Digital subtraction angiography (DSA) guided sequential sclerotherapy for maxillofacial vein malformation. Eur Rev Med Pharmacol Sci 2014;18:1709-1712

22. Orlando JL, Caldas JG, Campos HG, Nishinari K, Krutman M, Wolosker N. Ethanol sclerotherapy of head and neck venous malformations. Einstein (Sao Paulo) 2014;12:181-186

23. Ribeiro MC, de Mattos Camargo Grossmann S, do Amaral MBF, de Castro WH, Navarro TP, Procopio RJ, et al. Effectiveness and safety of foam sclerotherapy with $5 \%$ ethanolamine oleate in the treatment of low-flow venous malformations in the head and neck region: a case series. Int J Oral Maxillofac Surg 2018;47:900-907

24. Rosbe KW, Hess CP, Dowd CF, Frieden IJ. Masseteric venous malformations: diagnosis, treatment, and outcomes. Otolaryngol Head Neck Surg 2010;143:779-783

25. Sachin K, Rashmi S, Manish S, Siddhartha W, Uday L. Haemangiomas and venous malformations of the head and neck: a retrospective analysis of endovascular management in 358 patients. Indian J Plast Surg 2013;46:109-116

26. Shigematsu T, Sorscher M, Dier EC, Berenstein A. Bleomycin sclerotherapy for eyelid venous malformations as an alternative to surgery or laser therapy. J Neurointerv Surg 2019;11:57-61

27. Siniluoto TM, Svendsen PA, Wikholm GM, Fogdestam I, Edström S. Percutaneous sclerotherapy of venous malformations of the 
head and neck using sodium tetradecyl sulphate (sotradecol). Scand J Plast Reconstr Surg Hand Surg 1997;31:145-150

28. Songsaeng D, Churojana A, Khumthong R, Mahiwan L. Comparative outcomes for sclerotherapy of head and neck venous vascular malformation between alcohol and bleomycin. J Med Assoc Thai 2015;98:408-413

29. Spence J, Krings T, TerBrugge KG, Agid R. Percutaneous treatment of facial venous malformations: a matched comparison of alcohol and bleomycin sclerotherapy. Head Neck 2011;33:125130

30. Spence J, Krings T, terBrugge KG, da Costa LB, Agid R. Percutaneous sclerotherapy for facial venous malformations: subjective clinical and objective MR imaging follow-up results. AJNR Am J Neuroradio/ 2010;31:955-960

31. Stimpson P, Hewitt R, Barnacle A, Roebuck DJ, Hartley B. Sodium tetradecyl sulphate sclerotherapy for treating venous malformations of the oral and pharyngeal regions in children. Int $J$ Pediatr Otorhinolaryngol 2012;76:569-573

32. Su L, Fan X, Zheng L, Zheng J. Absolute ethanol sclerotherapy for venous malformations in the face and neck. J Oral Maxillofac Surg 2010;68:1622-1627

33. Wang D, Su L, Han Y, Wang Z, Zheng L, Li J, et al. Direct intralesional ethanol sclerotherapy of extensive venous malformations with oropharyngeal involvement after a temporary tracheotomy in the head and neck: initial results. Head Neck 2017;39:288-296

34. Wang YA, Zheng JW, Zhu HG, Ye WM, He Y, Zhang ZY. Sclerotherapy of voluminous venous malformation in head and neck with absolute ethanol under digital subtraction angiography guidance. Phlebology 2010;25:138-144

35. Zhao JH, Zhang WF, Zhao YF. Sclerotherapy of oral and facial venous malformations with use of pingyangmycin and/or sodium morrhuate. Int J Oral Maxillofac Surg 2004;33:463-466

36. Zheng JW, Yang XJ, Wang YA, He Y, Ye WM, Zhang ZY. Intralesional injection of pingyangmycin for vascular malformations in oral and maxillofacial regions: an evaluation of 297 consecutive patients. Oral Oncol 2009;45:872-876

37. Zhi K, Wen Y, Li L, Ren W. The role of intralesional Pingyangmycin in the treatment of venous malformation of facial and maxillary region. Int J Pediatr Otorhinolaryngol 2008;72:593-597

38. Luo QF, Gan YH. Pingyangmycin with triamcinolone acetonide effective for treatment of lymphatic malformations in the oral and maxillofacial region. J Craniomaxillofac Surg 2013;41:345349

39. Shiels WE 2nd, Kang DR, Murakami JW, Hogan MJ, Wiet GJ. Percutaneous treatment of lymphatic malformations. Otolaryngol Head Neck Surg 2009;141:219-224

40. Tu JH, Do HM, Patel V, Yeom KW, Teng JMC. Sclerotherapy for lymphatic malformations of the head and neck in the pediatric population. J Neurointerv Surg 2017;9:1023-1026

41. Flors L, Leiva-Salinas C, Maged IM, Norton PT, Matsumoto AH, Angle JF, et al. Mr imaging of soft-tissue vascular malformations: diagnosis, classification, and therapy follow-up. Radiographics 2011;31:1321-1340; discussion 1340-1341

42. Hage AN, Chick JFB, Srinivasa RN, Bundy JJ, Chauhan NR, Acord $M$, et al. Treatment of venous malformations: the data, where we are, and how it is done. Tech Vasc Interv Radiol 2018;21:45-54

43. Albanese G, Kondo KL. Pharmacology of sclerotherapy. Semin Intervent Radiol 2010;27:391-399

44. Yesil S, Tanyildiz HG, Bozkurt C, Cakmakci E, Sahin G. Single-center experience with sirolimus therapy for vascular malformations. Pediatr Hematol Oncol 2016;33:219-225

45. Klosterman T, O TM. The management of vascular malformations of the airway: natural history, investigations, medical, surgical and radiological management. Otolaryngol Clin North Am 2018;51:213-223

46. Sadick M, Wohlgemuth WA, Huelse R, Lange B, Henzler T, Schoenberg $\mathrm{SO}$, et al. Interdisciplinary management of head and neck vascular anomalies: clinical presentation, diagnostic findings and minimalinvasive therapies. Eur J Radiol Open 2017:4:63-68 